Itinéraires Itinéraires

Littérature, textes, cultures

\title{
Le corps nu féminin et la censure dans trois films japonais : la beauté des détours
}

Patrice Bougon

\section{OpenEdition}

Journals

Édition électronique

URL : http://journals.openedition.org/itineraires/1581

DOI : 10.4000/itineraires. 1581

ISSN : 2427-920X

Éditeur

Pléiade

Édition imprimée

Date de publication : 1 novembre 2011

Pagination : 189-200

ISBN : $978-2-296-55720-8$

ISSN : 2100-1340

Référence électronique

Patrice Bougon, "Le corps nu féminin et la censure dans trois films japonais : la beauté des détours ", Itinéraires [En ligne], 2011-3 | 2011, mis en ligne le 01 novembre 2011, consulté le 19 avril 2019. URL :

http://journals.openedition.org/itineraires/1581; DOI : 10.4000/itineraires.1581

\section{(ब) $(\Theta \Theta$}

Itinéraires est mis à disposition selon les termes de la licence Creative Commons Attribution - Pas d'Utilisation Commerciale - Pas de Modification 4.0 International. 


\title{
Le corps nu féminin et la censure dans trois films japonais : la beauté des détours
}

\begin{abstract}
Japanese culture can be considered as an art of the detour and ellipsis, according to Kûki Shûzo and Tanizaki Junichirô. The scope of this article is to analyse three films produced at a time when censorship was relatively strong. With Tales of the Moon and Rain (Ugetsu monogatari, 雨月物語) in 1953, by Mizoguchi Kenji, Manji (Svastika, ऋ) and Tatouage (Irezumi, 刺青) in 1966, by Masumura Yasuzo, we analyse how rhetoric of eclipse suggests thanks to several techniques what the audience have to imagine and that is not shown on the screen.
\end{abstract}

Keywords : movie, Japanese, rhetoric, erotic, adaptation

Mots clé : film, Japonais, rhétorique, érotique, adaptation

\section{Des récits aux films}

La culture japonaise peut être caractérisée comme celle du détour et de l'allusion, ce dont témoigne la communication la plus familière au Japon. Cette singularité culturelle donne à la littérature et aux films de ce pays, un supplément esthétique dont je vais donner quelques exemples, extraits de trois films tournés entre 1953 et 1966, c'est-à-dire produits à une époque encore soumise à une censure relativement stricte de la représentation du corps nu féminin. Avant d'analyser ces séquences, je solliciterai deux textes japonais qui pensent la spécificité de la beauté féminine au Japon. J'ai choisi des films qui sont des adaptations de grandes œuvres littéraires afin d'analyser comment deux arts différents travaillent la relation entre censure et représentation puisqu'à cette période, il était hors de question de montrer à un grand public plus que ce qu'autorisait le bon goût, mais aussi la censure de l'État nippon ${ }^{1}$.

1. La censure relative à la représentation du corps nu au Japon est assez paradoxale puisque la pilosité pubienne des femmes fut, jusqu'à une date récente, interdite à l'écran et même 
La comparaison entre représentation littéraire et représentation filmique sera aujourd'hui simplement esquissée ${ }^{2}$. Mon propos portera essentiellement sur la structure de trois films. J'ai choisi d'examiner l'adaptation par Mizoguchi Kenji des Contes de pluie et de lune (1776) d'Ueda Akinari, intitulée Les Contes de la lune vague après la pluie (1953), et les deux adaptations effectuées par Masumura Yasuzo de deux récits de Tanizaki Junichirô : Le Tatouage (1910), et Manji (1946). Mon projet général, dans lequel s'inscrit cette étude, est d'analyser comment les textes de ces auteurs japonais décrivent le corps nu féminin, tout en sollicitant, à son régime maximum, le travail de l'ellipse, de l'allusion, de la métonymie. En d'autres termes, lisant Ueda Akinari ${ }^{3}$ et Tanizaki Junichirô, j'interrogerai les relations entre lisible et illisible. J'ai choisi ces trois œuvres dans la mesure où il se trouve qu'elles ont été adaptées au cinéma et que la comparaison entre les deux formes de représentation donne à penser. L'usage du terme « adaptation » est problématique car la nouvelle de dix pages de Tanizaki - Irezumi - donne lieu à un film de quatre-vingt six minutes réalisé en 1966. Entre 1953 et 1966, la censure concernant les corps était encore très forte, mais celle-ci a eu aussi parfois un effet positif quant à la recherche formelle cinématographique qui, dès lors, a été incitée à inventer une rhétorique filmique qui montre, ou bien plutôt, évoque la nudité du corps féminin, par ellipses et éclipses. En d'autres termes, il s'agit ici d'analyser le rapport entre le visible et l'invisible. Mon hypothèse est que l'art cinématographique japonais montre peu et que le spectateur imagine beaucoup, pour suppléer ce qu'il ne perçoit pas sur l'écran, soit parce que la nudité est occultée par un arbre, un paravent ou un miroir, soit parce que le corps nu sort du cadre. Cette invisibilité du corps nu sur l'écran n'en rend pas moins fort l'érotisme filmique. L'analyse de courtes séquences va nous permettre d'indiquer par quels moyens purement filmiques (profondeur de champ, raccord-son, cadrage) l'imaginaire du spectateur est sollicité, voire excité. Le spectateur est, à la fois, frustré et jaloux car il constate l'effet du spectacle de la nudité dans le regard des personnages qui, eux, ont le plaisir de voir l'objet, pour lui invisible, de sa pulsion scopique, celle-là même, notons le, qui est l'un des fondements du plaisir du spectateur ${ }^{4}$.

sur des supports graphiques, alors que d'innombrables DVD mettant en scène des actrices jouant de très jeunes adolescentes torturées, exhibant des scènes excrémentielles sont autorisés. Tout semble permis, si le sexe est masqué par une mosaïque. Nous sommes donc loin du code Hays produit par le puritanisme protestant américain entre 1934 et 1966.

2. La présente étude est un chapitre d'un travail en cours portant essentiellement sur le nu féminin dans les films japonais des années 1960, mais qui portera aussi sur le corps nu masculin. J'analyserai en effet La Femme des sables d'Abe Kôbô (1962) et son adaptation filmique par Teshigahara Hiroshi en 1964.

3. Pour ce dernier, la tâche est impossible dans la mesure où Mizoguchi a très librement adapté deux nouvelles du recueil : Contes de pluie et de lune [trad. René Sieffert, Unesco, 1956], Paris, Gallimard, coll. « L'imaginaire », 2009.

4. Hitchcock met en scène cette pulsion dans Rear Windows (Fenêtre sur cour), 1956, film dont l'enjeu réflexif est essentiel. Truffaut qui a publié un livre d'entretien fondamental, 
Mais avant d'aborder ces séquences filmiques, il est utile de citer quelques phrases d'un essai - La Structure de l'iki ${ }^{5}$ (1933) - qui donnent une idée de ce qu'est, pour un esprit japonais du $\mathrm{Xx}^{\mathrm{e}}$ siècle, un corps féminin. L'auteur de cet essai se nomme Kûki Shûzô (1888-1941). C'est un philosophe qui a suivi les cours d'Husserl, d'Heidegger et qui eut pour répétiteur, en 1928, Jean-Paul Sartre. Dans son essai phénoménologique, le philosophe japonais évoque le charme qui émane du corps féminin légèrement voilé, exemple emblématique d'un certain type d'élégance qu'il nomme l'iki : «L'expression de l'iki est aussi perceptible lorsqu'un léger voile enveloppe entièrement la nudité » (p. 66).

La beauté du corps nu est donc une construction fantasmatique produite à partir de ce que donne à imaginer le voile, qui éclipse l'objet du désir. Un peu plus loin, la structure de l'iki est abordée dans son rapport à la temporalité :

Une autre attitude iki est visible lors de la sortie du bain. Le port d'un simple yukata d'après-bain sans apprêt, qui contient le souvenir de la nudité dans un passé proche, accomplit l'expression de l'attirance et sa cause formelle ${ }^{6}$.

Admirant une femme habillée d'un peignoir japonais, Kûki Shûzô peut imaginer une scène invisible antérieure dont la charge érotique est forte : le corps de la femme qui fut, un temps, nue dans le bain. Ce rapport au temps est actif dans l'art cinématographique par le travail du montage qui peut défaire la linéarité temporelle en engendrant des flash-back, mais aussi par des ellipses narratives permettant de couper la séquence pendant laquelle une femme se déshabille avant qu'elle soit montrée, en partie, derrière un élément du décor qui a aussi fonction de cache (miroir, paravent). Le cadre et ses caches participent donc aussi à l'ellipse du visible afin de donner à imaginer ce qui n'est pas représenté dans les limites de l'écran.

\section{Éloge du peu visible}

Il se trouve que Tanizaki, dont nous allons analyser deux adaptations filmiques, est aussi l'auteur d'un essai - Éloge de l'ombre $(1933)^{7}-$ qui propose une autre perspective, complémentaire de celle de Kûki Shûzô, pour penser le corps féminin japonais. Cet essai, essentiellement connu pour ses descriptions de l'ombre dans l'espace intérieur japonais, comporte aussi quelques pages peu commentées concernant le corps de la femme, du

Le Cinéma selon Hitchcock (Paris, Ramsay, 1966), a, par ailleurs, intitulé un recueil d'articles : Le Plaisir des yeux, Paris, Flammarion, 1987.

5. Trad. Camille Lovier, Paris, PUF, 1984.

6. Ibid.

7. Tanizaki Junichirô, Éloge de l'ombre [1933], Paris, Publications orientalistes de France, trad. René Sieffert, 1978. Rééd. Paris, Verdier, 2010. 
moins celui dont le modèle dominait encore à la fin du XIX ${ }^{\mathrm{e}}$ siècle. Toujours original, pour un esprit occidental dans sa manière d'aborder les choses, Tanizaki compare les femmes à des poupées traditionnelles :

Comme on le sait, au théâtre de bunraku les poupées féminines ne sont rien d'autre qu'une tête et des mains [...] j'estime pour ma part que ce procédé approche de très près la vérité, car les femmes d'autrefois n'avaient d'existence réelle qu'au-dessus du col et au bout des manches, le reste disparaissait entièrement dans l'obscurité. (p. 73)

Le corps de la femme de cette époque n'est donc qu'un support qui fait penser :

[...] irrésistiblement au bâton qui constitue l'armature des poupées. En vérité, le torse est alors un support destiné à recevoir le costume, et rien de plus. Ces femmes dont le torse est aussi réduit à l'état de support, elles sont faites d'une superposition de je ne sais combien d'épaisseurs de soie ou de coton, et si on les dépouillait de leurs vêtements, il ne resterait d'elles, comme pour les poupées, qu'un bâton ridiculement disproportionné. (p. 76)

Selon la même perspective, Tanizaki évoque quelques lignes plus loin « la beauté fantomatique de ces femmes-là ». Héritant de cette tradition, métamorphosée par son style singulier, le film de Mizoguchi met en scène ce caractère spectral de la femme, au sens propre et figuré, comme nous allons le voir, le personnage féminin se révélant un fantôme. Au Japon, le fantôme est un personnage présent dans de nombreux récits et films, mais aussi, encore de nos jours, dans la vie quotidienne ${ }^{8}$. D'une certaine façon, Les Contes de la lune vague après la pluie représente, par les moyens du film, cette analyse de Tanizaki :

Les manifestations de spectres ou de monstres n'étaient somme toute que des émanations de ces ténèbres, et les femmes qui vivaient en leur sein, entourées de je ne sais combien de rideaux écrans, de paravents, de cloisons mobiles, n'étaient-elles pas, elles-mêmes, de la famille des spectres? (p. 88)

Dans sa conclusion, Tanizaki établit une relation intéressante pour notre propos entre l'ombre, les femmes et la littérature : « J'aimerais élargir l'auvent de cet édifice qui a nom "littérature", en obscurcir les murs, plonger dans l'ombre ce qui est trop visible et en dépouiller l'intérieur de tout ornement superflu » (p. 103).

Les essais de Kûki Shûzô et de Tanizaki offrent un cadre théorique qui nous permet d'aborder trois séquences filmiques donnant à penser comment représenter et occulter le corps nu féminin selon trois styles différents.

8. Aujourd'hui encore, bien des Japonais, avant de se coucher, voilent les miroirs, officiellement pour les protéger de la poussière, mais aussi plus ou moins inconsciemment par peur du retour des fantômes. Pensons aussi, en France, aux films de Cocteau dans lesquels le miroir est une surface liquide qui fait office de porte entre les vivants et les morts. 


\section{Le charme spectral d'une femme fantomatique ${ }^{9}$}

Le film de Mizoguchi est l'histoire d'un pauvre potier installé dans un village qui va dans une ville pour vendre sur un marché sa modeste production afin de pouvoir nourrir sa femme et son enfant. Une femme, très élégante et mystérieuse, Dame Kutsuki, l'invite à livrer ses poteries chez elle. Espérant faire de bonnes affaires, il se rend dans la demeure de cette femme qui vit seule avec sa servante. D'emblée, la Dame Kutsuki cherche à séduire cet homme naïf qui est comme envoûté par cette femme dont on va comprendre peu à peu qu'elle est un fantôme. Après une nuit d'amour uniquement évoquée par le discours de Dame Kutsuki, la servante incite sa maîtresse à prendre un bain dans une source naturelle (onsen) avec celui qu'elle nomme d'emblée son «mari ». C'est cette séquence qui donne à voir l'évocation d'une nudité du corps féminin dont je voudrais montrer en quoi elle sollicite des moyens spécifiques à l'art cinématographique de Mizoguchi. Par un fondu enchaîné très subtil, Mizoguchi fait passer le spectateur de l'intimité d'une chambre à coucher à l'espace d'un bois. L'écran donne à voir les arbres, les branchages, les feuilles. Qu'estce qui se cache dans cette dense végétation filmée dans une demi-obscurité ? Le spectateur suit ce que présente à ses yeux ce travelling vers la gauche, il se demande vers quoi le réalisateur oriente son regard et où se trouvent les personnages. L'énigme est assez vite levée puisqu'on aperçoit en profondeur de champ, un bain naturel fumant où se trouve déjà le potier, tandis que Dame Kutsuki passe sensuellement un tissu sur le cou et le dos de l'homme. Cette scène où l'homme semble l'objet de tous les soins de l'amoureuse donne cependant à entendre : "Tu es une chose à moi » (Anata wa watashi no mono) [52.42]. Dame Kutsuki contourne le bord du bain et se dirige vers la caméra, c'est à-dire vers nous, elle place le tissu entre ses dents ${ }^{10}$ pour libérer ses mains qui commencent à dénouer l'une de ses ceintures de l'un de ses kimonos. D'un pas rapide, elle continue à se dévêtir au moment même où elle entre dans le bois, de sorte que son corps est d'emblée éclipsé par les branchages, puis elle sort du cadre par la droite une seconde après, la perspective de la caméra n'étant pas modifiée. Le spectateur imagine le corps invisible de cette femme qui se déshabille en marchant prestement afin de rejoindre nue son amant après cette trajectoire en forme de boucle. Le potier regarde avec ravissement Dame Kutsuki revenir, sans que le spectateur n'ait perçu sur l'écran la moindre partie de son corps. C'est un rire et le bruit de son corps plongeant dans le bassin, qui nous font imaginer une scène érotique puissante dont l'écran n'a parado-

9. Au sujet de Contes de la lune vague après la pluie, film de Mizoguchi Kenji, 1953, adaptation de Contes de pluie et de lune, d'Ueda Akinari, 1776, traduit par René Sieffert, [Éditions Unesco, 1956], Paris, Gallimard, coll. «L'imaginaire » (comportant le DVD), 2009.

10. Signalons que dans les estampes érotiques japonaises, il est fréquent que la femme fasse ce geste de séduction. 
xalement rien montré, si ce n'est l'ombre de la femme, une seconde avant d'entrer dans le bain. Le regard de jouissance du potier, et la bande son qui nous fait entendre off le rire de la femme, compensent l'absence de figuration des corps nus. Un dernier plan nous fait voir le potier qui sort du cadre sur la droite pour rejoindre le corps de Dame Kutsuki qui est hors champ visuel, un raccord-son nous fait cependant imaginer le début de l'étreinte.

\section{Avoir une femme dans la peau : Le Tatouage (Irezumi) ${ }^{11}$}

Dans Le Tatouage, nouvelle de Tanizaki de huit pages ${ }^{12}$, une jeune femme ${ }^{13}$ de 16 ou 17 ans, en formation chez une geisha, est porteuse d'une lettre qui demande à un tatoueur réputé - Seikichi - d'exercer son art sur sa peau « pour aider à la promouvoir » (p. 6) ${ }^{14}$.

Le tatoueur est d'emblée caractérisé par ce qu'un psychiatre nommerait deux perversions. D'une part, Seikichi est en effet décrit comme un homme ayant plaisir à faire souffrir, le narrateur évoquant que « des jouissances inconnues d'autrui étaient enfouies au plus profond du cœur du jeune maître », ce qu'il obtient par " l'insupportable supplice de ses aiguilles » (p. 4), d'autre part, tel un fétichiste, il est fasciné par le pied d'une femme ${ }^{15}$. Dans ce très court récit, presqu'une demie-page est en effet consacrée à la description du « pied nu de femme d'une blancheur de neige. Pour un œil aussi pénétrant que le sien, les pieds d'un être humain reflétaient autant que le visage tout un jeu d'expressions complexes » (p. 5). Deux pages plus loin, Seikichi indique pourquoi la jeune femme qui lui rend visite n'est pas vraiment une inconnue et en quoi la puissance de sa passion est purement visuelle : «— Voilà cinq longues années que je t'attends. C'est la première fois que je vois ton visage, mais de ton pied je me souviens bien...» (p. 7). Mais ce doux pervers est aussi présenté comme un artiste, un authentique peintre d'estampes déclassé. L'un des enjeux du récit est d'indiquer en quoi la contemplation de l'œuvre graphique méduse, au même titre que la contemplation du corps d'une femme, surtout si elle est endormie ${ }^{16}$.

Lorsque le tatoueur montre deux peintures représentant des femmes démoniaques de la mythologie japonaise, la jeune fille veut échapper à

11. Au sujet de : Le Tatonage (Irezumi) de Masumura Yasuzo, 1966, 86 minutes, adapté de Le Tatouage (Shisei), 1910, de Tanizaki Junichirô, 1910.

12. Notre édition de référence est celle de la collection «Bibliothèque de la Pléiade », t. I, Paris, Gallimard, 1997, p. 3-11, trad. de Marc Mécréant.

13. L'auteur n'indique pas son nom, manière d'en faire non pas un simple personnage mais une figure allégorique pour penser les rapports entre art, modèle, créateur, vie et mort.

14. Les notes du traducteur indiquent que la pratique de se faire tatouer au XIX ${ }^{\mathrm{e}}$ siècle était courante pour une geisha.

15. Tanizaki est l'auteur d'un autre récit dont le titre est Le Pied de Fumiko, 1919.

16. Sur ce thème, lire Kawabata Yasunari, Les Belles endormies [1961], trad. de René Sieffert, Paris, Albin Michel, 1970. 
ce spectacle qui lui montre son futur destin de femme fatale. Elle affirme sa peur, mais au lieu de fuir, elle se couche sur le ventre en masquant son visage, s'offrant ainsi d'une certaine façon à celui qu'elle craint. Le tatoueur en profite pour l'endormir avec une drogue et il contemple, lui aussi pétrifié, la beauté de ce corps ensommeillé. Notons, en mémoire d'Éloge de l'ombre, que la lumière est indirecte et qu'elle est tamisée par les fenêtres de papier :

Un soleil radieux frappait de plein fouet la surface du fleuve et semblait incendier la pièce de huit nattes réverbérées par le miroir des eaux, ses rayons irisaient d'ondes dorées le papier des cloisons coulissantes et l'innocent visage de la jeune endormie [...] Seikichi, son matériel à la main, resta un bon moment assis sans bouger sur les nattes, plongé dans le ravissement. (p. 9)

Dans le récit, rien n'est dit de la mise à nu et de son degré : on assiste directement au travail du tatoueur s'exerçant sur l' " épiderme virginal » (p. 9) du dos. L'acte de tatouer consiste ici à pénétrer le corps de la femme, la métaphore est surdéterminée ${ }^{17}:$ "Chaque goutte instillée de cinabre des Ryûkyû diluée dans l'alcool de riz était comme une goutte de sa propre vie; il y voyait la couleur des émois de son âme» (p. 9). À l'analogie sexuelle, s'ajoute des réflexions portant sur l'effet médusant de l'œuvre d'art, mais aussi sur le rapport mortifère entre l'œuvre et l'artiste. Une fois, le tatouage terminé, Seikichi : «[...] resta en contemplation devant l'araignée incrustée dans le dos de la jeune fille. Oui, toute sa vie avait passé dans ce tatouage [...] » (p. 10). Au réveil, consciente du pouvoir de séduction que va lui procurer cette araignée ${ }^{18}$, la jeune femme s'écrit : " Maître, faites-moi vite voir mon tatouage! » (p. 10). Seikichi la conduit dans la salle de bain : « [...] viens prendre un bain très chaud pour aviver les couleurs. Tu auras sûrement mal [...]» (p. 10-11). Le tatoueur voit donc le corps nu entrer dans l'eau de la baignoire, mais l'ellipse descriptive prive le lecteur du plaisir éprouvé par celui-ci dans la salle de bain : "Quand elle sortit du bain, elle n'avait même pas le courage de s'essuyer, mais écarta sans ménagement la main secourable de Seikichi » (p. 11).

Dans la version filmée, nous allons l'analyser, la censure et le déplacement s'effectuent selon d'autres figures. Notons enfin que quelques lignes avant la fin de la nouvelle, le narrateur tient à souligner que c'est moins la femme qui compte, comme objet de désir, que la figuration de l'araignée : «Avant de partir, laisse-moi voir encore une fois ton tatouage » (p. 11).

La logique de notre doux pervers est donc d'une grande rigueur dont le principe directeur est celui-ci : contrairement à l'orgasme, le plaisir visuel n'est pas limité dans le temps, ce dont témoigne aussi le plaisir du cinéphile.

17. Freud signale, dans une analyse de cas, l'équivalence produite par un patient, entre encre et sperme, ces deux liquides créateurs.

18. Au Japon, l'araignée est animal qui fait signe vers la prostitution, comme l'indique une note du traducteur. 
Dans le film Le Tatouage (Irezumi) de Masumura Yasuzo, il s'agit moins d'une adaptation que d'une variante puisque le réalisateur a transformé une nouvelle de huit pages en un film de quatre-vingt six minutes ${ }^{19}$. Je ne commenterai que la scène du début du film qui correspond à celle du récit de Tanizaki dans la mesure où il est intéressant d'analyser comment l'érotique filmique invente ses moyens propres pour évoquer une nudité suggérée autrement par l'art littéraire de cet écrivain singulier.

Le film s'ouvre sur une scène ${ }^{20}$ de tatouage qui est suivie par vingtcinq minutes de flash-back, racontant ce qui s'est passé auparavant avant de reprendre encore cinq minutes et de s'achever sur la contemplation du tatoueur, un peu effrayé par l'araignée qu'il vient de représenter.

Le premier plan est celui d'un visage de femme éclairé par une bougie placée dans une lanterne portable tenue par un homme dont on n'aperçoit que le bas du corps. La pièce est obscure, la femme allongée sur le sol, est liée par une large ceinture rouge de kimono. Elle tente de s'éloigner de l'homme mais elle ne peut que ramper et rouler sur elle-même dans sa vaine fuite. Le spectateur s'attend à un viol, mais si l'homme fera effectivement preuve de violence, il n'y aura pas pour autant acte sexuel. Ce n'est pas la femme en tant que telle qui l'intéresse (le spectateur le comprendra peu à peu) mais la qualité exceptionnelle de la peau considérée uniquement comme support d'un tatouage. Ce qui est remarquable dans cette séquence, c'est la façon dont sont cadrés et montés les différents plans de sorte que l'apparente scène de viol est sublimée par un travail sur la couleur et l'ambiguïté des plans. Au premier plan du visage [0.15] qui exprime la peur, succède une série de plans où l'alternance des couleurs transforme cette représentation réaliste en une figuration abstraite puisque ce qui nous est donné à voir en un premier temps, ce n'est pas le corps, mais les différentes couleurs des trois kimonos qui la recouvrent.

L'écran est en effet totalement occupé par le rouge du bas du premier kimono [0.22], puis par le jaune d'un second, le visage étant alors horscadre. La femme roule, dans la pénombre, et l'on aperçoit les trois couches de kimonos. Peu de temps après, elle s'assied. Après cette longue séquence muette (de près d'une minute) ouvrant le film, les premières paroles sont enfin énoncées. Dès lors, le spectateur comprend que l'homme n'a pas l'intention de violer cette femme. Seikichi fait part de son admiration ( « ta peau parfaite ») puis il endort la femme à l'aide d'une drogue [1.38]. Elle est alors manipulée comme un simple paquet dont l'homme défait

19. Pour des raisons de place, je n'aborde pas l'ensemble des transformations opérées par Masumura, j'y reviendrai ailleurs, car elles ont l'intérêt de proposer une interprétation de la nouvelle.

20. Notre édition de référence est le DVD distribué par Night and Day. Notre séquence se déroule de 15 secondes à 4 minutes et 10 secondes. Nous indiquerons ainsi entre crochets les références temporelles des plans. 
fébrilement les trois enveloppes (kimonos) de couleurs différentes, chacune étant fermée par une ceinture de tissu. À nouveau, l'écran ne montre qu'un jeu de couleurs, le visage de la femme étant hors cadre. Au rouge succède, le jaune, les carreaux gris et blanc, à nouveau, le rouge [1.58]. Un plan montre le bas du ventre et le rouge d'une sorte de jupon. Ce plan isolé peut faire croire à une mise à nue éminente ${ }^{21}$, mais les mains de l'homme (dont depuis le début du déshabillage le visage est hors-cadre) décident, sans hésitation, de retourner le corps désirable, afin de découvrir le dos, encore protégé par un kimono jaune. L'objet du désir est enfin visible, cette surface de travail - la peau - dont la main du tatoueur apprécie, en spécialiste, la finesse du grain [2.24]. Quelques secondes après [2.31], Masumura sollicite la profondeur de champ, afin de placer, caméra au sol, au premier plan ${ }^{22}$, les instruments du tatoueur (plusieurs encriers, pinceaux et stylets), mais aussi les mains qui s'activent à préparer l'encre.

En second plan, la femme sous l'effet de la drogue n'est qu'à demie endormie sur le ventre, son visage posé sur un traversin, elle se retourne vers le spectateur qui a pu admirer son dos, ses épaules et sa nuque. L'ambiguiité de la situation est amplifiée par Masumura qui, par le jeu du cadrage et du montage, érotise une séquence qui ne l'est pas tellement dans la nouvelle de Tanizaki. Par un montage alterné [notamment en 3.25 et 4.01], montrant en gros plan, successivement, d'une part, le travail du tatoueur pénétrant la peau et l'avancée de la figuration de l'araignée, et d'autre part, un gros plan de la femme s'agrippant à son traversin en gémissant de douleur, Masumura sollicite, en le métamorphosant, un stéréotype des films japonais érotiques où souffrance et jouissance féminines sont représentées comme équivalentes. Lorsque la caméra revient en gros plan sur le travail des mains du tatoueur, le gémissement de douleur a alors pris une signification érotique supplémentaire. Le raccord-son produit dès lors une continuité avec le plan précédent où l'on voyait le visage de la femme souffrant et/ou jouissant. Dans la reprise finale de cette scène qui dure elle aussi, à peu près cinq minutes [25-30 minutes], poursuivant ce que le flash back a interrompu pendant vingt-cinq minutes, le tatoueur après une nuit de travail contemple son œuvre achevée. Un plan montre l'étrangeté du rapport entre la femme (dont la peau n'est que le support de l'œuvre), la représentation de l'araignée, et le tatoueur qui est son créateur. En effet, se réveillant peu à peu et respirant fortement, après toute une nuit de souffrance, la femme remue peu à peu son buste de sorte que l'image tatouée bouge, l'araignée dont les longues pattes enserre la totalité du dos de la femme est comme vivante.

21. Masumura joue sur la frustration du spectateur excité par cette sorte de strip-tease, à ce moment détourné de son but et qui a aussi l'intérêt de respecter la censure et la décence d'un film de 1966.

22. Manière spécifiquement cinématographique d'indiquer que ce qui importe, c'est moins la femme comme objet de désir que le travail de l'artiste. 
On notera enfin que deux miroirs sont nécessaires pour que la femme puisse, de façon doublement indirecte, admirer son tatouage et que, contrairement au texte de Tanizaki, le tatoueur n'accompagne pas la femme dans la salle de bain. Celle-ci enlace son tatoueur-bourreau de façon ambivalente, un plan montrant les bras enserrant l'homme, et un autre les pattes de l'araignée menaçante prête à enserrer ses futures proies : des hommes dont le tatoueur réalise qu'il sera l'un d'eux.

\section{Aimer son corps : Passion (Manji) ${ }^{23}$}

Une femme mal mariée - Sonoko - s'ennuie et suit des cours de dessin dans une école d'art fréquentée uniquement par des femmes. Un jour, elle dessine un modèle nu, légèrement voilé, représentant la déesse de la miséricorde $^{24}$. Le directeur de l'établissement lui fait remarquer que, malgré son talent habituel, le dessin est peu ressemblant. Elle s'aperçoit alors qu'elle a représenté, à son insu, le corps d'une autre élève - Mitsuko - dont elle est inconsciemment amoureuse. Montrant à Mitsuko son dessin, celle-ci le trouve un peu inexact quant aux formes de son corps. Sonoko lui demande de faire le modèle pour améliorer ce dessin. Mitsuko, après une hésitation feinte, profitant de l'absence du mari, se rend avec Sonoko, dans la chambre du couple pour jouer au modèle et finir dans les bras de son amie ${ }^{25}$. Cette séquence [11.40-16.36] nous permet d'analyser la dialectique entre le visible et l'invisible qui la structure, en étant attentif à deux objets du décor : le paravent et le miroir qui ont fonction de cache.

Le film s'ouvre par des gros plans montrant des sculptures de femmes nues, éclairées de telle façon, qu'en un premier temps, le spectateur peut croire voir des corps de femmes réelles. Ces plans initiaux en annoncent d'autres qui reviennent dans notre séquence et qui, cette fois, donneront à voir les corps nus des deux amies. Une première fois, celui de Mitsuko, puis une seconde fois, ceux enlacés des deux femmes. Dans tous les plans, remarquons que le visage est, soit caché, soit hors cadre, c'est aussi le cas pour les images des sculptures de sorte que le référent est, un temps, mis entre parenthèses pour nous placer face à un ensemble un peu abstrait de courbes, présentées en gros plan, c'est-à-dire dans un espace insituable tout en jouant sur les relations entre les zones d'ombre et de lumière.

Notre séquence commence [10.59] par l'entrée de Mitsuko et de Sonoko qui arrive directement dans la chambre. D'emblée, la perspective

23. Au sujet de Passion de Masumura Yasuzo, 1964, 91 minutes, adapté de Manji [19281930] de Tanizaki Junichirô, trad. de René de Ceccatty et Ryôji Nakamura, Paris, Gallimard, 1985, coll. « Folio », 1988.

24. Le modèle figure donc un être divin presque nu, assez sensuel qui préfigure le rapport de Sonoko à Mitsuko, mélange de vénération et de désir érotique.

25. Pour des raisons de place, je n'analyserai pas en détail le spectacle de cette union à la fois charnelle et abstraite. J'y reviendrai ailleurs. 
est signifiante dans la mesure où le cadre de la porte dans lequel apparaissent les deux femmes est lui-même cadré par un paravent, placé devant la caméra, qui ne laisse voir que la partie supérieure du buste de Mitsuko. Le paravent, plus qu'un simple élément du décor, est l'un des éléments centraux de la mise en scène de cette séquence. Les deux jeunes femmes passent devant le paravent qui sépare la chambre, proprement dite, d'un lavabo. Le lieu de la rencontre - la chambre d'un couple - est qualifié, à plusieurs reprises, de « sacré26 $"$, tout comme l'est le corps supposé vierge de Mitsuko. Ces expressions, énoncées par les actrices, sont des citations du roman. Cet adjectif fait donc de cette simple scène de pose et de séduction, une cérémonie. Avant de se mettre nue, Mitsuko demande à Sonoko de fermer les rideaux ${ }^{27}$, celle-ci se retourne pour effectuer ce geste [11.57], quelques secondes après [12.02] un son fait pour elle événement : elle entend le bruit de la robe que Mitsuko enlève. Sonoko ne peut s'empêcher de se retourner aussitôt pour admirer le corps dénudé de son amie. Quant au spectateur, placé de l'autre côté du paravent, il ne voit qu'une métonymie de cette nudité, en l'occurrence, le rapide dépôt de la robe sur le haut du paravent. Le spectateur ne perçoit rien du corps dénudé puisque la moitié droite de l'écran est occupée par le paravent. Seul le regard fasciné de Sonoko nous indique l'effet érotique de ce spectacle.

Pendant toute cette séquence, la caméra ne cesse d'aller d'un côté et de l'autre de ce paravent qui a donc statut de cache et qui a pour fonction d'exciter et de frustrer le désir de voyeur du spectateur. Quelques plans suivants, la caméra est placée derrière Sonoko qui observe Mitsuko enveloppée dans un drap blanc, celle-ci se dirige vers la gauche et dévoile, sans le vouloir (?), son dos tout en maintenant d'une main le drap sur ses hanches. Elle retire le tissu qui recouvre le miroir, comme souvent, au Japon ${ }^{28}$. Mitsuko s'admire dans le miroir [12.32], bientôt rejointe par Sonoko qui commence à perdre le contrôle d'elle-même lorsque son amie pivote et laisse bailler le drap pour dévoiler un peu plus ses formes. Sonoko s'agenouille alors pour mieux l'admirer, excitée par tant d'érotisme dont le spectateur ne contemple qu'une faible partie. Sonoko ordonne : « Je veux en voir plus [...] enlève ce drap » [13.20-13.44]. Folle de désir, Sonoko tente d'arracher cette pièce de tissu qui finit par se déchirer de sorte que le spectateur voit enfin Mitsuko nue, mais accroupie [13.53]. Elle a le visage posé sur le matelas, si bien que le spectateur est, à nouveau, face à un corps, sans visage. Avec la moitié de drap qui lui reste entre les mains Mitsuko se relève et se place devant le miroir qui, durant toute cette séquence, aura eu une double fonction contradictoire : montrer le reflet cadré du corps mais aussi le cacher au spectateur puisque la caméra se place alternativement,

26. En japonais : Shinsei

27. Il s'agit, non pas de tissu, mais de fenêtres coulissantes de papier mat - shôji - dont parle Tanizaki dans Éloge de l'ombre.

28. Nous avons évoqué ici même la fonction de ce voile dans la note 8 . 
d'un côté et de l'autre, du miroir. Une étape érotique supplémentaire est franchie lorsque Sonoko réussit, avec l'assentiment réticent de Mitsuko, à arracher le reste du drap [14.45], le spectateur la voit alors totalement nue, là encore visage caché, cette fois-ci, par ses cheveux, une main masquant son sexe, l'autre, une partie de ses seins. Un travelling vertical ascendant donne à admirer ce corps, selon un savant mélange de zones d'ombre et de lumière, qui fait rime visuelle avec les plans de sculptures, situés à l'ouverture du film, dont nous avons parlés en introduction. Le regard de Sonoko fait le trajet inverse, son corps peu à peu s'agenouillant, ses yeux, telle une caméra, descendent vers le bas du corps de Mitsuko, de sorte que dans cette position, elle se trouve au niveau du sexe de son amie, protégé par ses mains pudiques. Le regard du spectateur qui a d'abord fait avec elle ce travelling vertical descendant est maintenant orienté par une nouvelle perspective choisie par le cinéaste qui a placé la caméra derrière Mitsuko, de sorte que le spectateur voit le visage de Sonoko, exultant de désir lorsqu'elle écarte peu à peu les mains de son amie afin de contempler son sexe [15.05]. Là encore le spectateur qui ne perçoit pas le résultat de ce geste imagine ce qui ne peut être montré sur l'écran.

Par l'analyse de trois séquences montrant des corps nus féminins japonais, nous avons démontré comment trois films très différents inventent, chacun à sa manière, une poétique spécifiquement cinématographique afin de jouer avec le désir du spectateur. Ainsi le son peut-il produire des images mentales chez le spectateur, confronté à une rhétorique filmique de l'ellipse qui permet dans le même temps de montrer et de cacher le corps nu féminin. Sans doute l'esthétique japonaise traditionnelle, que nous avons évoquée en introduction, a-t-elle préparé cette culture à inventer des détours spécifiquement cinématographiques quant à l'art de suggérer la beauté du nu féminin. 